\title{
The Multiscale Stiffness of Electrospun Substrates and Aspects of their Mechanical Biocompatibility
}

\author{
Manuel Zündel ${ }^{\mathrm{a}}$, Alexander E. Ehreta ${ }^{\mathrm{a}, \mathrm{b}}$, Edoardo Mazza ${ }^{\mathrm{a}, \mathrm{b}, *}$ \\ ${ }^{a}$ ETH Zürich, Institute for Mechanical Systems, 8092 Zürich, Switzerland \\ ${ }^{b}$ Empa, Swiss Federal Laboratories for Materials Science and Technology, 8600 Dübendorf, Switzerland
}

\begin{abstract}
In contrast to homogeneous materials, the mechanical properties of fibrous substrates depend on the probing lengthscale. This suggests that cells feel very different mechanical cues than expected from the macroscale characterisation of the substrate materials. By means of multiscale computational analyses we study here the mechanical environment of cells adhering to typical electrospun networks used in biomedical applications, with comparable macroscopic stiffness but different fibre diameters. The stiffness evaluated at the level of focal adhesions varies significantly, and the overall magnitude is strongly affected by the fibre diameter. The microscopic stiffness evaluated at cell scale depends substantially on the network topology and is about one order of magnitude lower than the macroscopic stiffness of the substrate, and two to three orders of magnitude below the fibres' elastic modulus. Moreover, the translation of stiffness over the scales is modulated by global deformations of the scaffold. In particular, uniaxial or biaxial stretching of the substrate induces nonlinear microscopic stiffening. Finally, although electrospun networks allow long-range transmission of cell-induced deformations, the comparison between the range of forces measured in cell traction force microscopy and those required to markedly deform typical electrospun networks reveals an order of magnitude difference, suggesting that these scaffolds provide a rather rigid environment for cells. All these results underline that the achievement of mechanical biocompatibility at all relevant lengthscales, and over the whole range of physiological loading states is extremely challenging. At the same time, the study shows that the diameter, length and curvature of fibre segments might be tunable towards achieving this goal.
\end{abstract}

Keywords: Electrospun networks, Tissue engineering, Cell substrates, Multiscale stiffness, Computational modelling

\section{Introduction}

The ability of the electrospinning process to handle a large variety of fibre base materials and to control multiple topological parameters allows manufacturing fibrous networks within a wide design space $[1,2]$. This quality made electrospun networks (ESNs) potential candidates for multifarious applications, amongst others in biomedical research and regenerative medicine [3-5], where the micro-fibrous and network-like structure of ESNs bears the potential to mimic the morphology of the extracellular matrix (ECM) [6]. Significant amounts of research have therefore been dedicated to ESNs as scaffolds for tissue engineering of, e.g., skin [7], cardiac [8] and vascular [9] grafts. Such grafts aim at substituting defective native tissues with an engineered equivalent, i.e. a living construct of a synthetic scaffold and native cells [10-12]. The scaffold needs to functionally substitute the native ECM, and would thus ideally provide the same chemical, biological and, finally, mechanical environment for the cells (see e.g. Vacanti et al. [13]). It is therefore the scaffold's ability to satisfy these different aspects of biocompatibility that will finally be decisive for its interaction with the surrounding cells and tissues $[14,15]$, and finally determine the success of the graft.

The different aspects that govern the mechanical biocompatibility of the scaffold [16] can mainly be related to two distinct length scales [17]: Macroscopically, the mechanical biocompatibility is associated with the necessity of compatible deformations between the engineered scaffold and the surrounding host tissues [16, 17], typically characterised

*Corresponding author (mazza@imes.mavt.ethz.ch)

Preprint submitted to -

This document is the accepted manuscript version of the following article:

October 10, 2018

Zünde1, M., Ehret, A. E., \& Mazza, E. (2019). The multiscale stiffness of electrospun substrates and

aspects of their mechanical biocompatibility. Acta Biomaterialia, 84, 146-158.

https://doi.org/10.1016/j.actbio.2018.11.012

This manuscript version is made available under the CC-BY-NC-ND 4.0 1icense http://

creativecommons.org/1icenses/by-nc-nd/4.0/ 
by non-linear, time dependent and anisotropic characteristics [18]. Further macroscopic requirements originate from functional aspects, i.e. from the functions that the engineered construct takes over from the native (replaced) tissue, such as load carrying capabilities, adequate strength and maintaining tissue integrity. Microscopic requirements arise since the scaffold defines the immediate environment of the cells living within the engineered construct. At first instance, this implies prerequisites on scaffold morphology, e.g., with respect to pore size and shape, and the availability of sites that allow and promote cellular adhesion [19]. In addition to these morphological and purely static aspects, the requirements on this length scale $(\sim \mu \mathrm{m})$ relate to the sensitivity of cells to mechanical loads, perceived through their mechanotransductive apparatus [20]. More precisely, the need to provide a suitable mechanical microenvironment constrains the admissible deformations of such scaffolds at fibre length scale, experienced by the cells as motions, forces and structural stiffness at their focal adhesions (FAs) [17].

The complex multidisciplinary and multiscale task of scaffold design is therefore key for the development of successful tissue engineered implants. While the suitability of a graft is unequivocally revealed during the in-vivo clinical evaluation, it can be useful to assess its biomechanical compatibility already during the design phase, based on quantitative design criteria. Following the biomimetic approach, such design criteria might be established from a direct comparison with native soft tissues [17]. The macroscopic requirements are accessible through the well established experimental methods for soft tissue mechanical characterisation. The identification of quantitative benchmarks at cellular length scale, however, is more intricate as it requires experimental techniques to probe the tissue at $\mu \mathrm{m}$ length scale, e.g., by micro-indentation methods [21-23] or analysis of local microscopic strain fields by imaging techniques $[24,25]$. The available results generally suggest that soft tissues are governed by peculiar, typically nonaffine micromechanics, which strongly differ from the ones expected for homogeneous materials. This concerns, for example, attenuation and amplification effects when strain is transferred over the scales [24-27], the long-range transmission of cell-induced deformations [28], and stiffness parameters that vary over multiple orders of magnitude with the probing length scale [29].

Additional cell-related requirements can be retrieved from mechanobiological assays and in-vitro studies with synthetic substrates [30]. For example, studies with cells cultivated on homogeneous flat substrates allow quantifying cell-exerted forces by means of traction force microscopy [31-33], and elucidated a large number of mechanosensitive pathways [34, 35]. This includes, e.g., the cell's sensitivity to surrounding stiffness and its gradients [36-38], topography [39, 40], and to mechanical loads [41-44]. Compared to the numerous studies performed on homogeneous continuous elastomer or hydrogel substrates, much less is known on the impact of the cell-scale stiffness in electrospun networks. Notwithstanding, information on beneficial or adverse morphologies of fibrous scaffolds has been derived from mechanobiological studies and assays (cf. [45]). Several studies have shown a strong dependence of cell shape, spreading, differentiation and proliferation on electrospun fibre diameter [46-50]. Fibre orientation was shown to affect mainly the shape and orientation of cells, which align and elongate in the direction of the fibres $[51,52]$. Other critical morphological parameters in ESN are the network porosity and pore size, which mainly influence cell proliferation and infiltration, the latter being particularly high if pores and cells are of comparable size [53]. By modifying the stiffness of the fibre material while maintaining topology, multiple studies revealed significant changes in the cell phenotype expression, differentiation and proliferation [54-56]. Since this modification impacts directly the stiffness felt by the adhering cells, these results suggest the latter as a particularly relevant parameter with regard to the mechanobiology of cells adhering to ESNs.

In view of the difficulties associated with the experimental characterization of the cellular microenvironment in ESNs, computational models may provide a valuable alternative towards understanding some key aspects. The potential of numerical models for such investigations has been previously showcased in studies on biopolymer networks, which investigated the transmission of strain through the scales [57, 58], the long-range transmission of deformation [59-61] and the stiffness sensing of cells [62]. In this work, an ESN-specific [63] computational model is employed to investigate aspects of mechanical biocompatibility of typical ESNs for biomedical use. Specifically, stiffness and kinematics at cellular length scale are investigated, and compared to the mechanical behaviour at macroscopic length scale. By this means parameters are identified that characterise the translation of these mechanical properties over the scales, and how this depends on network topology and macroscopic loading state. Through numerical simulations, this contribution thus provides qualitative and quantitative information on multi-scale properties of ESNs that may be essential for their mechanical biocompatibility, but are hardly accessible in experiments. In combination with experimental data on native tissues, this information can be used to rationalise the performance and, eventually, improve the design of ESNs as scaffolds for tissue engineering. 


\section{Methods}

\subsection{Multiscale simulation framework for electrospun networks}

The multiscale mechanical analysis was performed using a previously presented 2.5D discrete network model specific to electrospun networks (cf. Zündel et al. [63]). Briefly, representative volume elements (RVEs) of networks made of very long and planarily arranged fibres are generated using a custom Python (2.7, Python Software Foundation, OR, USA) algorithm. This algorithm accounts intrinsically for the layered disposition of fibres with limited out-of-plane reach typical for networks produced by electrospinning, and allows a physically motivated identification of cross-links, i.e. of the locations where fibres interact mechanically with each other. The mechanical analysis of the generated network geometries is performed using a commercial finite element software (Abaqus, Version 6.10-EF1, Dassault Systèmes Simulia Corp., Providence, RI, USA). To this end, the fibres are discretized with sequences of beam elements (element type B22), while the cross-links are implemented by coupling all degrees of freedom in the intersection location of the two fibres. Macroscopic deformation states are imposed on the RVE through the application of homogeneous or periodic boundary conditions on the networks boundary, and the problem is solved through implicit finite element analysis. While this gives access to the microscopic deformation patterns of the fibres within the RVE, the macroscopic homogenised stress tensor of the RVE is computed from the resulting force vectors at the boundary nodes (cf. [64-66]).

In particular, network RVEs with normalized dimensions of $25 \bar{l}_{\mathrm{S}} \times 25 \bar{l}_{\mathrm{S}} \times 10 t_{\mathrm{I}}$ were considered for the prediction of the macroscopic response (Fig. 1b), where $\bar{l}_{\mathrm{S}}$ and $t_{\mathrm{I}}$ indicate the average segment length and the interaction thickness, respectively [63]. Periodic boundary conditions were defined to represent uniaxial tension with nominal tensile strain $\varepsilon_{1}$, and the tensile nominal (first Piola-Kirchhoff) stress $P_{1}$ was computed. As a measure of homogenized stiffness at macroscopic scale, the elastic modulus $E^{\text {macro }}$ was estimated from the secant of the stress-strain response to uniaxial tension $\left(P_{1}\right.$ vs. $\varepsilon_{1}$, Fig. 1 b) at $\varepsilon_{1}=0.5 \%$.

\subsection{Modelled electrospun networks}

In this study, two electrospun scaffolds with different network characteristics were considered. Specifically, the analysed networks were set-up to represent networks of poly- $\epsilon$-caprolactone (PCL) fibres with diameters of $d_{\mathrm{F}}=$ $0.3 \mu \mathrm{m}$ and $d_{\mathrm{F}}=1 \mu \mathrm{m}$ respectively, representative for previous mechanobiological and tissue engineering studies presented in literature (cf. [67]). Although changes in fibre diameter are typically accompanied by alterations in network porosity [68], different means have been discussed to change the latter independently [68, 69]. Thus, due to the lack of a unique relation between fibre diameter $d_{\mathrm{F}}$ and porosity $\Phi$, the latter was set to the same value of $\Phi=90 \%$ for both networks, which is representative for PCL ESNs with diameters in the range considered here, cf. [70-72]. The other network parameters were varied with fibre diameter $d_{\mathrm{F}}$ (Tab. 1). For a given $d_{\mathrm{F}}$, the average curvature radius $\bar{r}$ and the average segment length $\bar{l}_{\mathrm{S}}$ were defined from the experimental relations determined by Pai et al. [73], and the fibre material's Young's modulus was selected according to the results of single PCL fibre tensile tests reported by Wong et al. [74], assuming linear elastic and incompressible material behaviour (fibre Poisson's ratio $v^{\text {fibre }}=0.5$ ).

For the analysed morphologies (Fig. 1a) and fibre material laws, the model predicts very similar macroscopic behaviour for the two network types. Subjected to uniaxial tension, the networks exhibit a fairly linear stress response with strong lateral contraction (Fig. 1b). Macroscopic elastic moduli of the ESNs were determined as $E^{\text {macro }}=$ $4.04 \mathrm{MPa}$ and $E^{\mathrm{macro}}=4.33 \mathrm{MPa}$ for fibre diameters of $d_{\mathrm{F}}=0.3 \mu \mathrm{m}$ and $d_{\mathrm{F}}=1 \mu \mathrm{m}$, respectively. The linearised stiffness values of the analysed networks are therefore representative for the lower end of the wide range of tensile stiffness commonly reported for soft biological tissues (2-560MPa [29]). Similarly, in the analysed deformation range

\begin{tabular}{|l|l|l|l|l|l|}
\hline$d_{\mathrm{F}}[\mu \mathrm{m}]$ & $\bar{r}[\mu \mathrm{m}]$ & $\bar{l}_{\mathrm{S}}[\mu \mathrm{m}]$ & $\Phi[-]$ & $E^{\text {fibre }}[\mathrm{MPa}]$ & $v^{\text {fibre }}[-]$ \\
\hline 0.3 & $46^{\dagger}$ & 15.2 & 0.9 & 332.3 & 0.5 \\
\hline 1.0 & $173^{\dagger}$ & 4.56 & 0.9 & 258.6 & 0.5 \\
\hline
\end{tabular}

Table 1: Topographical parameters used for the generation of the ESN. ${ }^{\dagger}$ Fibres have been assumed to be sinus-shaped, and the according shape parameters (amplitude and wavelength) were selected to match the given average curvature radii. 
a)

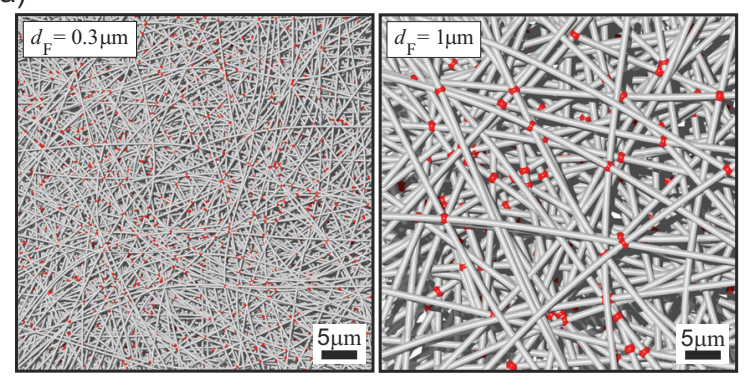

b)

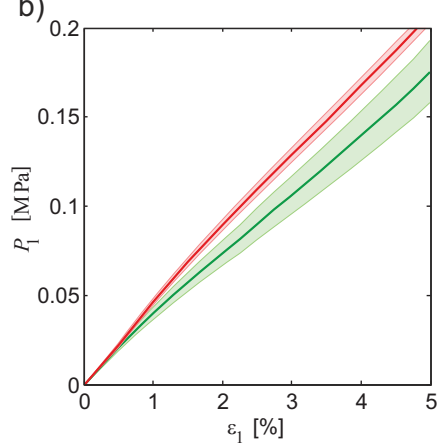

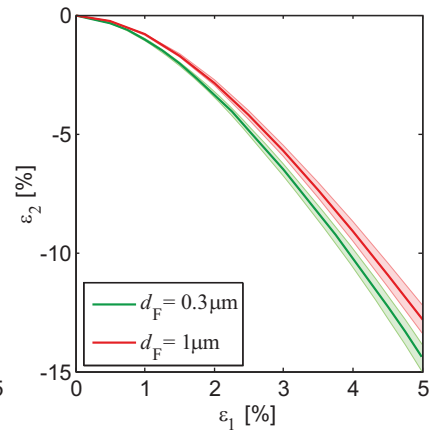

Figure 1: a) Graphical visualization of the networks analysed in this study with $d_{\mathrm{F}}=0.3 \mu \mathrm{m}$ and $d_{\mathrm{F}}=1 \mu \mathrm{m}$. Red symbols indicate the locations of fibre cross-links. b) Macroscopic response of the considered networks to uniaxial tension, reported as nominal stress (left) and lateral contraction (right) response to tensile strain $\varepsilon_{1}$. Results are reported as mean and standard deviation (shaded areas) of $N=5$ networks.

the networks feature incremental Poisson ratios (cf. [75]) between 2 and 5, comparable to the values reported for soft tissues, e.g., Glisson's capsule [76], ligaments [77] and tendons [78].

\subsection{Modelling of cell-network interactions}

To study the interaction between cell and network the former was strongly simplified, accounting mainly for the attachment sites of the cell to the substrate, i.e. the focal adhesions, and the contractile capability of the stress fibres of the cytoskeleton. The model assumes a quasi-circular, non-polarized cell which adheres to the top surface of the modelled networks at its centre, which coincides with the origin of the coordinate system (Fig. 2a). For simplicity, the cell's adhesion sites to the network (i.e. the focal adhesions) were placed with constant distance from the cell centre and therefore lie on circle, here with $30 \mu \mathrm{m}$ diameter (Fig. 2ab). Experimental observations reported in literature suggest that cells with comparable spread area typically have between 20 and 100 focal adhesions [79]. Considering that focal adhesions which are very close to each other might adhere to the same fibre and therefore can be considered as a single interaction location, a total of 24 interaction sites between cell and network (i.e. single focal adhesions or clusters) were assumed. These interaction sites were identified based on the projected intersection of the circular pattern with the fibres nearest to the upper network surface (Fig. 2b). The involved fibres were partitioned at these locations and remeshed, allowing a localized tie coupling of the fibres with the stress fibres of the cell model. The latter were discretized using connector elements that link mechanically each contact site with the centre of the cell (Fig. 2c). Modelled as linear actuators, these connector elements can either generate cell contraction when the connector
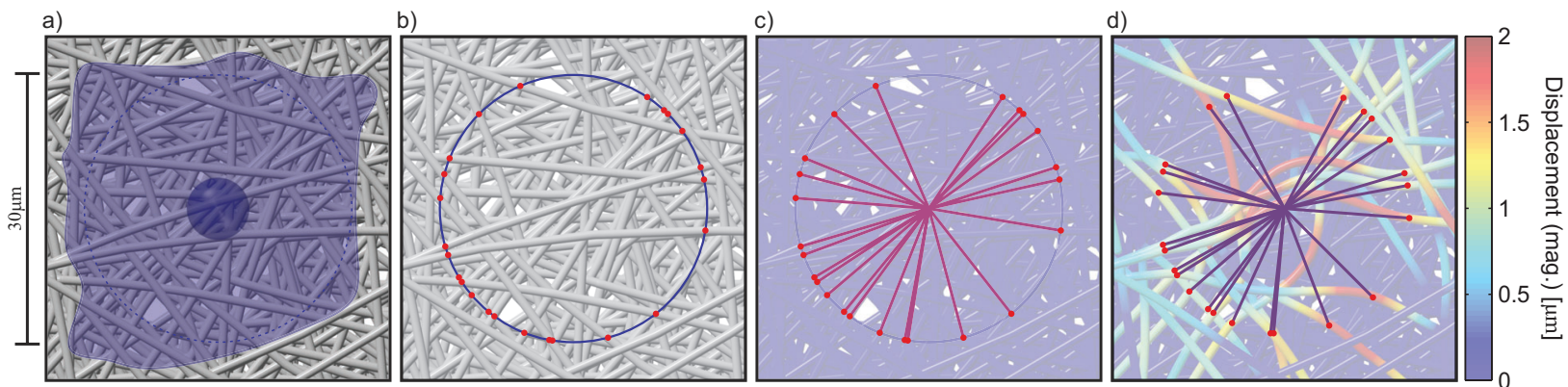

Figure 2: Schematic representation of the modelled interaction of a cell adhering to the networks surface (a). First the intersections of cell edge with the nearest fibres were identified (b), allowing the successive coupling of fibres with a connector network representing the function of the cell's cytoskeleton (c). d) Contraction of connector network for the simulation of homogeneous cell contraction. 
lengths are reduced through a prescribed contraction, or stretch passively in absence of such prescribed conditions to accommodate the displacements induced at the attachment sites when the network deforms (Fig. 2d). The forces acting in the connector elements due to imposed contraction were extracted and provide the force vectors exerted by the individual focal adhesions. The stiffness that cells feel at their attachment sites is of structural origin and was approximated for each focal adhesion $i$ by the secant of the force displacement response

$$
k_{i}^{\text {micro }}=\frac{\left\|\Delta \vec{f}^{\vec{\imath}}\right\|}{\left\|\Delta \vec{u} \cdot \vec{e}_{f}^{i}\right\|},
$$

where $\vec{e}_{f}^{i}$ indicates the unit vector in direction of the force $\Delta \vec{f} \vec{t}$, which is oriented in radial direction towards the cell centre (Fig. 3a). The RVEs used for the analysis of cell-network interactions were selected large enough $(300 \mu \mathrm{m})$ to assure negligible influence of the RVE boundaries on the cell (see Appendix B). During cellular contraction the fibre nodes on the lateral network boundaries were fixed, whereas macroscopic network deformation was imposed with homogeneous boundary conditions if required (see Sec. 2.6).

\subsection{Analysis of cell interactions with homogeneous isotropic substrates}

To compare the cell-network interaction with the case of cells adhering to highly deformable, homogeneous and continuous substrates, analogous continuum finite element simulations were performed. The interaction of cell and substrate was modelled with 24 attachment sites to the network (focal adhesions) of elliptic shape $(2 \mu \mathrm{m} \times 1 \mu \mathrm{m})$ [80] spaced regularly on a circular pattern (diameter $30 \mu \mathrm{m}$ ) and pulling towards the centre with traction forces homogeneously distributed within each focal adhesion (Fig. A.1a in Appendix A). The substrate is modelled as a nearly incompressible hyperelastic body using the neo-Hookean material law provided by the finite element software with Poisson's ratio $v=0.4995$ and shear modulus adjusted to match the linearised stiffness $E^{\text {macro }}$ of the analysed networks (Sec. 2.2). For the numerical solution, the substrate geometry was discretized with a very fine mesh (characteristic element size $0.1 \mu \mathrm{m}$, see Fig. A.1b in Appendix A) in the region of the focal adhesions, and the degrees of freedom of the lateral boundaries were fixed. The force exerted by each focal adhesion is given by the multiplication of area with applied traction stress, whereas the corresponding displacement was evaluated as the average displacement of all finite element mesh nodes within the focal adhesion (Fig. A.1c in Appendix A). For comparison with the network simulations (see Sec. 2.5), the applied traction force was modulated to generate either an equivalent focal adhesion displacement $u_{\mathrm{FA}}$ or cell contraction $\varepsilon^{\mathrm{A}}$ (Eq. 2).

\subsection{Evaluations at cellular length scale and multiscale stiffness factor}

At the level of a whole cell, the contraction can be defined through the change of the projected area $A^{\text {cell }}$ of the polygon spanned by the attachment sites, allowing the quantification of cell contraction through the areal strain

$$
\varepsilon^{\mathrm{A}}=\frac{A_{0}^{\text {cell }}-A^{\text {cell }}}{A_{0}^{\text {cell }}} .
$$

In the simulations, the work $W^{\text {cell }}$ spent by the cell to achieve this contraction on an ESN is given by the sum of the work, i.e. the integral of axial force over elongation, of the individual cell connectors (Fig. 2c).

We define the multiscale stiffness factor $\Gamma$, which relates the linearised macroscopic stiffness $E^{\text {macro }}$ of an ESN to a stiffness exhibited by the material at cell level, comparable to an equivalent homogeneous isotropic substrate with $E^{\text {equiv }}$. This relation can be expressed as

$$
E^{\text {equiv }}=\Gamma E^{\text {macro }}
$$

where $\Gamma<1$ indicates a softer microscopic response than expected from the macroscopic response. Considering the difficulties associated with the definition of a material stiffness at microscopic lengthscale, we propose here to compute $\Gamma$ by comparing the work $W^{\text {cell }}$ with the energy $W_{\text {hom }}^{\text {cell }}$ computed for an equivalent contraction $\varepsilon^{\mathrm{A}}$ on a homogeneous substrate (dashed lines in Fig. 6a), therefore approximating the multiscale stiffness factor as

$$
\Gamma\left(\varepsilon^{\mathrm{A}}\right)=\frac{W^{\text {cell }}\left(\varepsilon^{\mathrm{A}}\right)}{W_{\text {hom }}^{\text {cell }}\left(\varepsilon^{\mathrm{A}}\right)} .
$$


To study the influence of fibre diameter $d_{\mathrm{F}}$, mean segment length $\bar{l}_{\mathrm{S}}$, and average curvature radius $\bar{r}$ on $E^{\text {macro }}, E^{\text {equiv }}$ and $\Gamma$ (Sec. 3.3.1), these topological parameters were varied from 20 to $250 \%$ of their original value, using the ESN with $d_{\mathrm{F} 0}=1.0 \mu \mathrm{m}$ as a reference and keeping the porosity constant $(\Phi=90 \%)$.

\subsection{Analysis of the influence of macroscopic deformations on cell-scale stiffness}

Under the in-vivo loading conditions to which tissue engineered grafts are subjected, the scaffold undergoes macroscopic deformations. These deformations are associated with local changes of network morphology that may affect the stiffness experienced at cell scale. To study this, the network RVEs were deformed by imposing homogeneous boundary conditions prior to the multiscale stiffness analysis described in Sec. 2.5. In particular, uniaxial tension and equibiaxial extension up to $10 \%$ strain were applied to the networks prior cell contraction (Fig. 3bc). Subsequently cellular contractions of $\varepsilon^{\mathrm{A}}=10 \%$ were imposed with respect to the new (deformed) configuration of the cell, for which the cell centre was placed in the centre of the 24 adhesion sites (Fig. 3d).
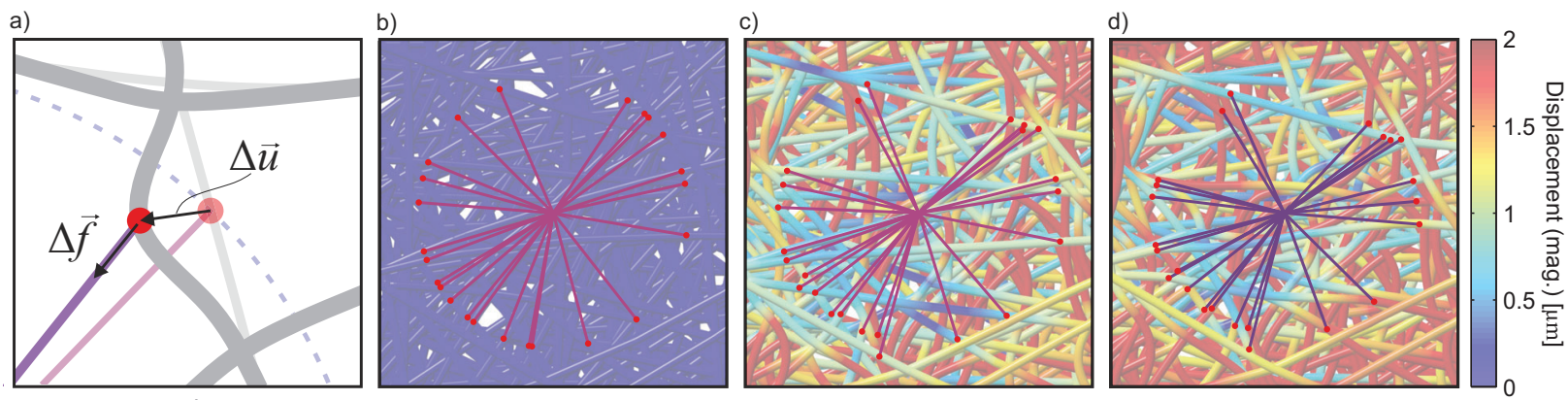

Figure 3: a) Schematic representation of the force and displacement vectors at focal adhesion level. Schematic representation of the modelled cell-network interaction for macroscopically imposed deformation: b) Adhesion sites and cell-connectors on the undeformed network, after macroscopic uniaxial tensile deformation (c) and subsequent connector contraction (d).

\subsection{Statistical Methods}

All numerical simulations were performed multiple times ( $\mathrm{N}=5-50$, as describe in the respective figure legends) to account for the randomness of the modelled networks. Unless otherwise specified, all quantitative data is presented as mean $( \pm)$ standard deviation which were computed with Matlab (Version 2013a, TheMathWorks Inc., Natick, MA, USA). No statistical analysis was performed to characterize significance.

\section{Results}

\subsection{Network kinematics at cellular length scale}

The analysis of the displacement field induced by the contraction of an adherent cell, reported quantitatively and qualitatively (in the inset) in Fig. 4a, reveals that the cell induced perturbation decays slowly in radial direction and exhibits significant spatial heterogeneity, as reflected by the high variability of the displacement magnitude. Comparison of the spatial decay observed in the ESNs and on the homogeneous substrate reveals substantial differences in the kinematics at cellular length scale: Compared to homogeneous substrates, both analysed ESNs exhibit markedly different displacement patterns, associated with a transmission of the cell-induced substrate deformations over a significantly larger range (Fig. 4b). 
a)

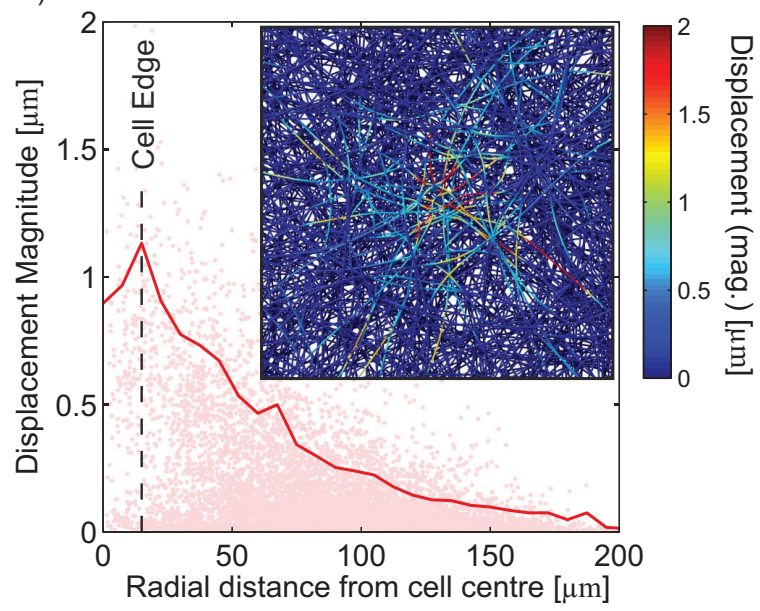

b)

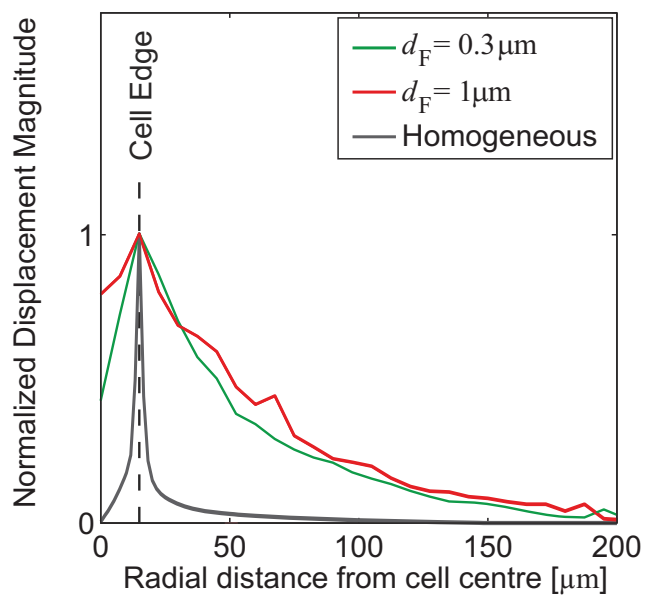

Figure 4: a) Cross-link displacements (shaded markers) induced by contracting cells for focal adhesion displacements of $u_{\mathrm{FA}}=1.0 \mu \mathrm{m}$ on ESN with $d_{\mathrm{F}}=1.0 \mu \mathrm{m}$. Data is reported for $N=10$ networks, and the solid line marks the upper bound of the $95 \%$ interval. The color bar decodes the fibre displacement magnitudes of the cell-deformed networks reported in the inset. b) Comparison of the upper bound of the $95 \%$ interval of the displacement fields induced by the cell on the ESNs and the homogeneous solid substrate. To facilitate the comparison, the curves were normalized by their respective value at the cell edge.

\subsection{Network stiffness at cellular length scale}

The results presented in the previous section are purely based on kinematics and independent of network stiffness, representing only one aspect of the micromechanical environment. The ability of cells to induce such deformations, however, depends strongly on the compliance of the substrate. Therefore this section presents a detailed investigation of the stiffness experienced by cells adhering to the two ESNs of comparable macroscopic stiffness.

\subsubsection{Stiffness heterogeneity at the level of focal adhesions}

Evaluations of the cell-scale stiffness for the two considered ESNs are reported in Fig. 5ab for FA displacements of $0.1 \mu \mathrm{m}$ and $1 \mu \mathrm{m}$, showing that the microstructural stiffness $k^{\text {micro }}$ is extremely scattered in both network types, thus

a)

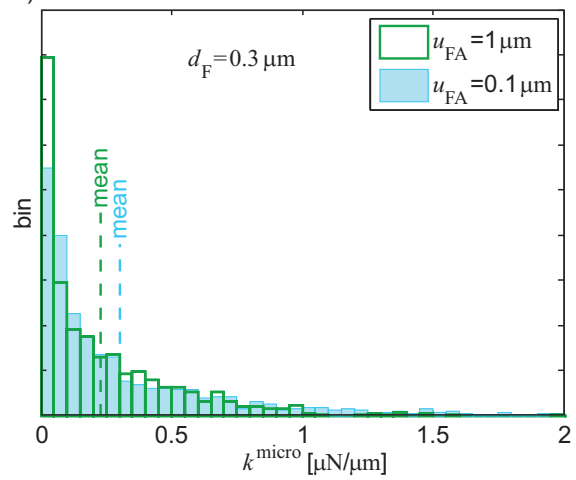

b)

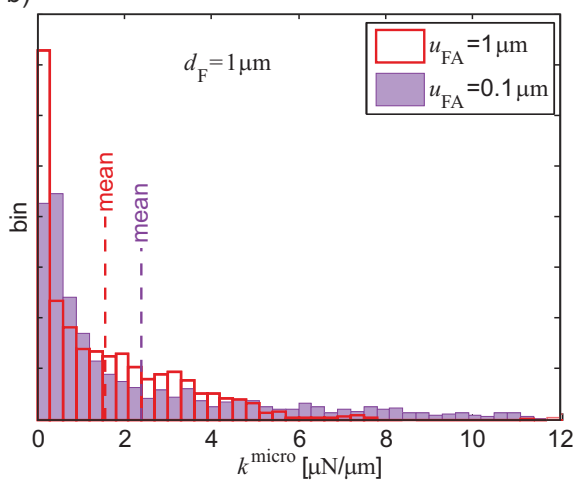

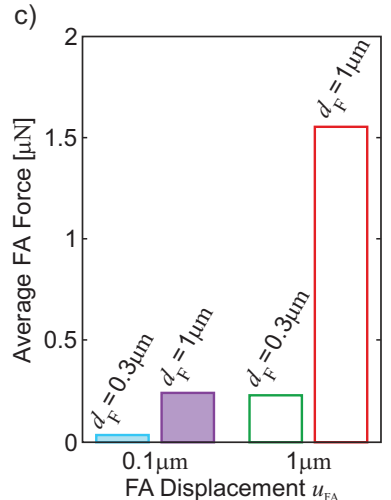

Figure 5: a,b) Distributions of secant stiffnesses felt by cells on ESN at focal adhesion level for $u_{\mathrm{FA}}=0.1 \mu \mathrm{m}$ and $u_{\mathrm{FA}}=1.0 \mu \mathrm{m}$, respectively on networks with $d_{\mathrm{F}}=0.3 \mu \mathrm{m}$ (a) and $d_{\mathrm{F}}=1.0 \mu \mathrm{m}$ (b). c) Average FA forces exerted by cells reported in function of FA displacement magnitude and ESN fibre diameter. Data is reported for $N=50$ simulations each (1200 FAs). 
strongly heterogeneous. Interestingly, the comparison of averaged stiffness values suggests that both ESNs exhibit a non-linear response to deformation at the level of focal adhesions, resulting in a softer response for increasing cell contraction. In terms of stiffness magnitudes, the two networks are very different: The force required to induce the same focal adhesion displacements on the networks with $d_{\mathrm{F}}=0.3 \mu \mathrm{m}$ is, on average, 7 to 8 times lower than on the networks with thicker fibres (Fig. 5c), despite the nearly identical mechanical response exhibited by the two ESNs at the macroscopic scale (Fig. 1b).

\subsubsection{Work as a measure of stiffness at whole cell level}

The evaluation of cell work for both network types reported in Fig. 6a shows again the significantly softer micromechanical environment provided by the ESN with thinner fibres. Additionally, the comparison with Fig. 5ab shows that the evaluation at cellular length scale for $N=50$ cells results in much lower variability at this than at FA length scale. This indicates that the mechanical heterogeneities have a characteristic length smaller than the spatial extension of the cell (i.e. cell diameter), leading to mechanical cues with significanty reduced variability when averaged over the cell.
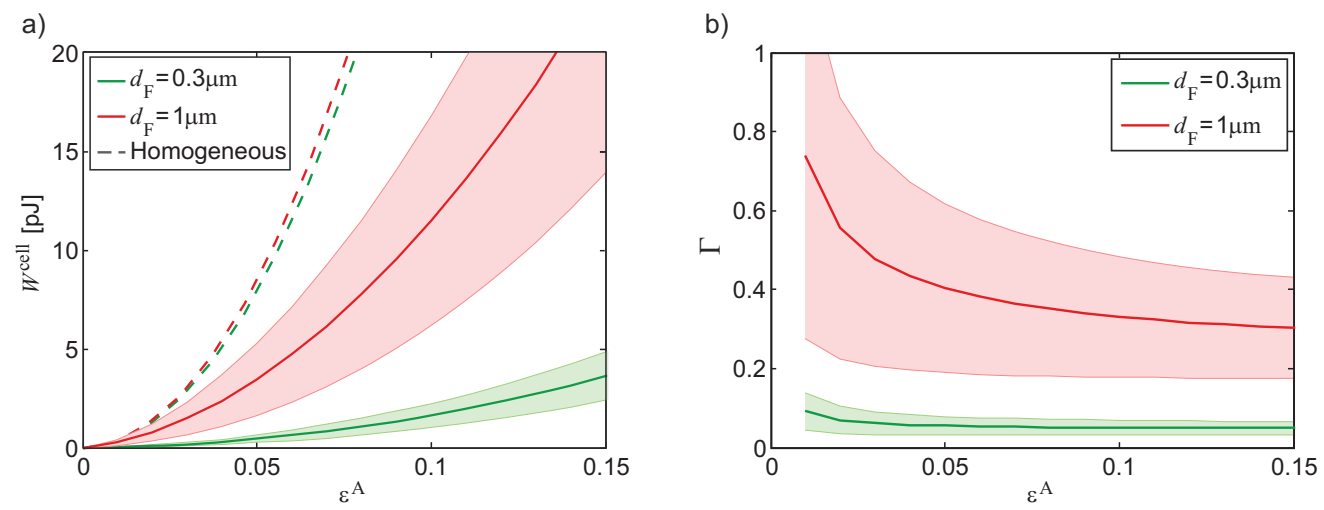

Figure 6: a) Cellular work needed for contraction on ESN, reported as mean (solid line) and standard deviation (shaded area) for $N=50$ cells on ESNs with $d_{\mathrm{F}}=0.3 \mu \mathrm{m}$ (green) and $d_{\mathrm{F}}=1.0 \mu \mathrm{m}$ (red). Dashed lines indicate the cellular work needed on homogeneous substrates with macroscopic stiffness (elastic modulus) equal to the analysed ESNs. b) Multiscale stiffness factor $\Gamma\left(\varepsilon^{\mathrm{A}}\right)$, reported as mean (solid line) and standard deviation (shaded area) for $N=50$ cells on ESNs with $d_{\mathrm{F}}=0.3 \mu \mathrm{m}$ (green) and $d_{\mathrm{F}}=1.0 \mu \mathrm{m}$ (red).

\subsection{The topological decoupling of stiffness}

The previously defined microstructural stiffness $k^{\text {micro }}$ might be suited for the comparison of networks at microscopic scale, but difficult to relate to their macroscopic response, which is needed to compare the network's mechanical characteristics at different scales. Evidently such scale dependence is not present in continuous homogeneous substrates, for which macroscopic stiffness and that experienced by the cells coincide. To compare fibrous and homogeneous substrates the work $\left(W_{\text {hom }}^{\text {cell }}\right.$ ) exerted by contracting cells on the latter was used as benchmark as described in Sec. 2.5, and the multiscale stiffness factor $\Gamma$ (Eq. 4) was calculated. To this end, the material properties of the homogeneous substrates were adapted so that their linearised response to uniaxial tension matched the response of the analysed ESNs, i.e. both types of substrates had identical $E^{\text {macro }}$. The values of $\Gamma$ (Fig. $6 \mathrm{~b}$ ) indicate that for a cellular contraction $\varepsilon^{\mathrm{A}}=0.1$, the ESNs with $d_{\mathrm{F}}=1.0 \mu \mathrm{m}$ feel three times softer to cells than a macroscopically equivalent homogeneous substrate, while for $d_{\mathrm{F}}=0.3 \mu \mathrm{m}$ they feel even 20 times more compliant.

\subsubsection{The dependence of multiscale stiffness on topological parameters}

The results presented in Fig. 7 highlight the different, length scale dependent impact of the topological parameters $d_{\mathrm{F}}, \bar{l}_{\mathrm{S}}$ and $r$ on the multiscale stiffness. A strong dependence of $\Gamma$ on fibre diameter is observable (Fig. 7c), which results from a negligible effect of $d_{\mathrm{F}}$ on $E^{\text {macro }}$ in contrast to a strong impact on the stiffness at cellular length 
a)

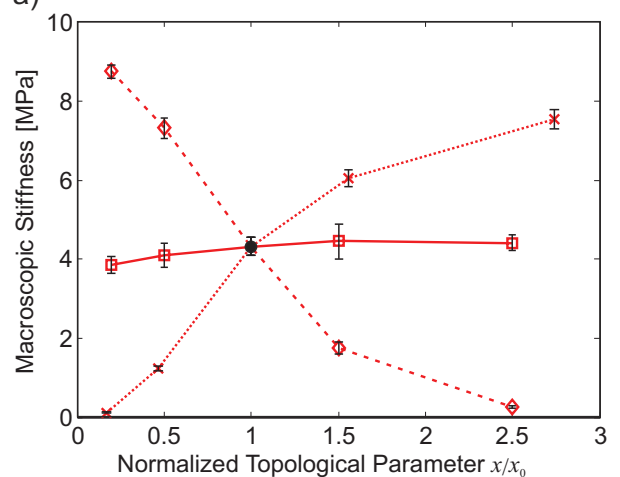

c)

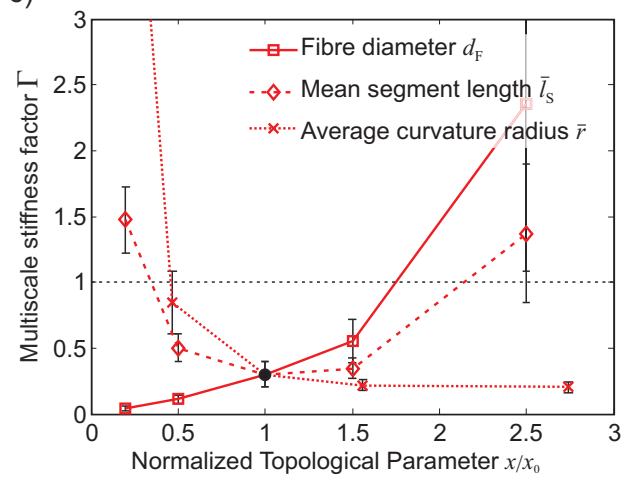

b)

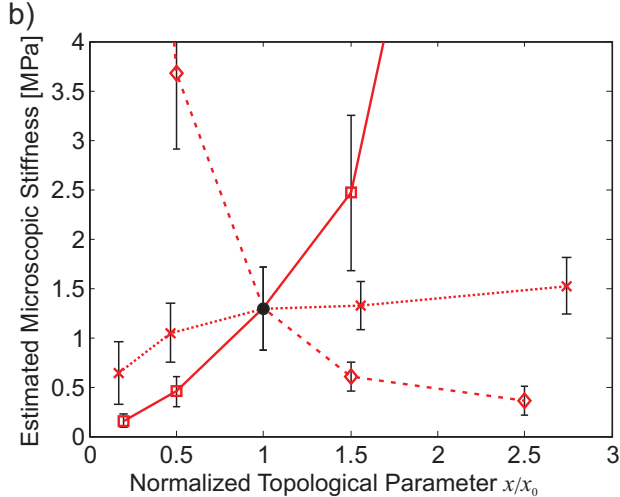

d)

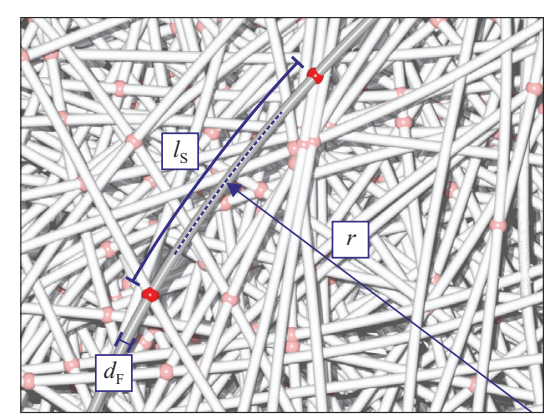

Figure 7: Sensitivity of the macroscopic stiffness (a), the estimated microscopic stiffness (b) and the multiscale stiffness factor (c) to the variation of topological parameters, which are schematically depicted in (d). The previously presented ESN with $d_{\mathrm{F}}=1.0 \mu \mathrm{m}$ was used as reference. Data is reported as mean and standard deviation for $N=10$ networks each.

scale $\left(E^{\text {equiv }}\right)$, eventually resulting in microscopically softer networks with decreasing diameter. The influence of fibre curvature is opposite, mainly because higher fibre curvatures (i.e. small curvature radii) results in an initially softer homogenised response, and therefore in a lower elastic modulus at macroscopic scale, while the microscopic response is less influenced. Similarly, very long average segment lengths seem to have a stronger impact on macroscopic than on microscopic stiffness, therefore leading to increasing values of $\Gamma$.

\subsection{External deformation induced microscopic stiffening}

The analysis of cell-scale stiffness for uni- and equibiaxially pre-stretched networks reveals that the multiscale stiffness factor can change significantly with type and magnitude of the applied macroscopic deformation (Fig. 8). Specifically, the results highlight that equibiaxial stretching alters the microscopic stiffness, with a substantial increase of $\Gamma$ already for a few $\%$ of macroscopic deformation, while for larger deformations $\left(\lambda^{\text {macro }}>1.05\right)$ the value of $\Gamma$ stabilizes for both network types (Fig. 8). The ESNs with $d_{\mathrm{F}}=1.0 \mu \mathrm{m}$ exhibit stronger microscopic stiffening induced through macroscopic extension and, contrary to the networks with $d_{\mathrm{F}}=0.3 \mu \mathrm{m}$, also uniaxial pre-stretching has a significant effect. Noteworthy for the $d_{\mathrm{F}}=1.0 \mu \mathrm{m}$-fibre network, a moderate equibiaxial extension turns the stiffness ratio from a $50 \%$ attenuation to a $50 \%$ amplification (Fig. 8b). We remark that the microscopic stiffening caused by macroscopic loads cannot be predicted, or even foreseen, from the macroscopic response, which is fairly linear for both analysed ESNs (Fig. 1b). 

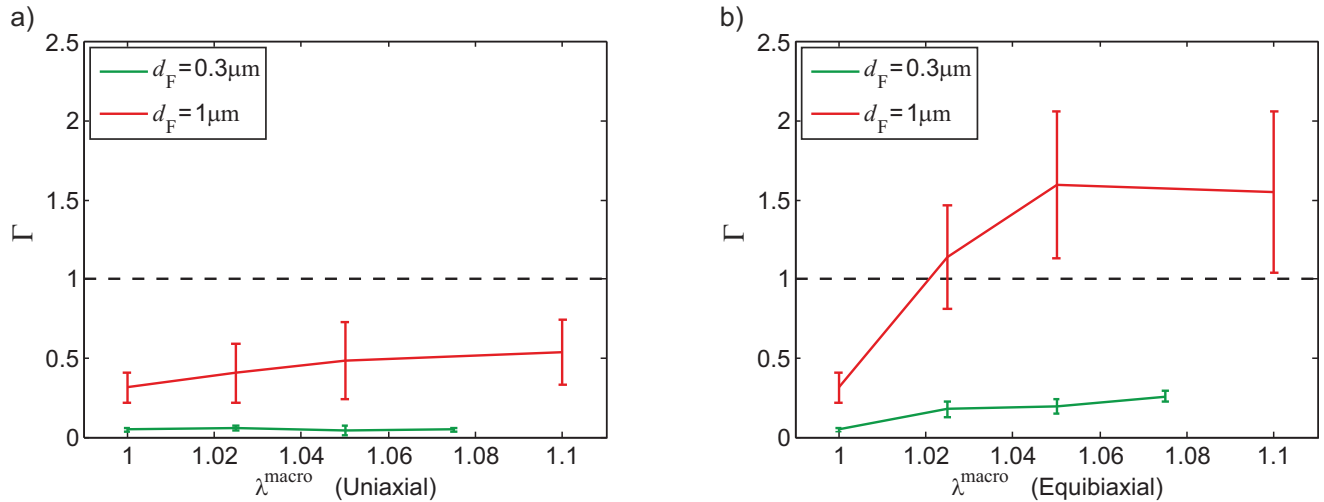

Figure 8: Relative microscopic stiffness of macroscopically pre-stretched networks with uniaxial (a) and equibiaxial tensile loadings (b) evaluated at $\varepsilon^{\mathrm{A}}=10 \%$. Data is reported as mean and standard deviation for $N=10$ networks.

\section{Discussion}

\subsection{The mechanical environment of the cell}

In line with experimental observations [56], the computational results in Sec. 3.1 (Fig. 4) suggest that ESNs allow for long-range transmission of cell induced deformations similarly as observed in biopolymer networks (cf. [81, 82]), and even with similar characteristic length scales [81]. The analysed networks feature a nearly linear stress-strain response, suggesting that the ability to transfer locally induced displacements over long distances originates in the fibrous nature of the material rather than from a non-linear macroscopic response as previously suggested (cf. [81, 82]). In fact, it might be the non-linear response of the single fibres itself, given by their low compressive resistance and high tensile stiffness (once straightened) that allows passing on displacements over wide ranges within the considered ESNs. Since pulled fibres act as stiff rods in an otherwise compliant environment, in which fibre segments that are cross-linked to the cell-pulled fibres simply rotate to accommodate cross-link displacements, cell-induced network deformations can be transferred radially over wide distances.

Interestingly, the long-range transmission of deformations induced by cells is in opposition to the very narrow nearfield of defects observed in both natural and synthetic fibre networks. In fact, corresponding investigations suggest that the strain amplification induced by the 'singularity' decays very rapidly with increasing distance from the crack tip, so that a significantly smaller region is affected by the presence of the defect than in homogeneous materials [83, 84]. A possible explanation for these contrasting observations is given by the strongly load-case dependent kinematics of fibrous networks. Contrary to the cell-induced displacements studied here, that lead to a biaxial extension dominated state around the cell, the near field of defects is characterized by a quasi-uniaxial tension state close to the free edges of the defect, where fibres can easily reorient towards the loading direction. Due to their non-linear response this causes significant lateral contraction, leading to a compaction of the fibre network around the defect, which shields the network from the stress concentration [84], and limits the defect-induced perturbations in the displacement field to a close region.

The quantitative analysis of the force magnitudes necessary to induce marked displacements at the adhesion sites in the two analysed networks (Fig. 5) suggests that these forces are higher than the magnitudes typically reported for cells in literature (up to 80nN/FA) [85], especially for the networks with $d_{\mathrm{F}}=1 \mu \mathrm{m}$. This indicates that these ESNs provide a rather stiff micro-environment, at least on average. However, the large spatial heterogeneity of the stiffness at focal adhesion scale limits the significance of these averaged data for the characterization of the micromechanical environment, which might be better represented by the cell work. Also, the average stiffness at focal adhesion level neglects that the network might offer sufficient alternative adhesion sites with higher compliance, to which cells might actively change over by detaching and reattaching their focal adhesions. While it is generally expected that the heterogeneity of the environment affects the behaviour of cells [86], the particular impact of these heterogeneities requires a detailed consideration of the particular mechanotransductive pathways. Irrespectively, a question of general relevance is whether cells are more sensitive to the individual signals from the FAs and their intracellular variations, 
or to their average. For mechanical signals translated into biochemical signals directly at the adhesion sites through protein-protein interactions (cf. [87, 88]), this key question is difficult to answer from a mechanical point of view. However, at least the transfer of loads through the cytoskeleton and the associated deformation of the nucleus [89], which is associated with mechanosensitive capabilities [90], might point a high relevance of cell-averaged deformation sensing.

\subsection{Decoupling between microscopic and macroscopic properties}

The marked differences in microscopic stiffness (Fig. 5) and cellular work (Fig. 6) between the two networks with fibres of 0.3 or $1 \mu \mathrm{m}$ diameter highlight that, similarly to other structured materials, the mechanical properties in ESNs depend significantly on the probing length scale. Consequently, the assessment of macroscopic stiffness can only provide little information on the mechanical stimuli experienced by cells. More information might be contained in the lateral contraction, which is known to be strongly affected by the micro-kinematics of fibrous networks [84, 91], since the latter are controlled by topological parameters that affect fibre slenderness, such as fibre diameter or crosslink density, which have also a strong impact on the multiscale stiffness (Fig. 7). Furthermore, the results show that fibre topology, not their material properties, governs these differences, since a modification of the fibres' material stiffness $E^{\text {fibre }}$ itself is expected to scale both micro- and macroscopic stiffness in a proportional manner, thus leaving the multiscale stiffness factor (Eq. 3) unaffected for all strain levels. The decoupling of mechanical properties can also be observed by comparing the stiffness of the individual fibres to the stiffness of the whole network. For the networks analysed in this study, the ratio $E^{\text {fibre }} / E^{\text {macro }}$ is in the range of 60 to 80 , which is in line with experimental results reported in literature (cf. [92, 93] (macroscopic) and [74] (single fibre)). Since the porosity of the networks $(\Phi=90 \%)$ can explain only a factor of 10 based on the mixture rule, a non-negligible part of the stiffness ratio $E^{\text {fibre }} / E^{\text {macro }}$ must originate in the non-affine micro-mechanics of the networks. Together with $\Gamma=E^{\text {micro }} / E^{\text {macro }}$ in the order of $10^{-1}$ (Sec. 3.3), this indicates factors around $10^{3}$ between $E^{\text {micro }}$ and $E^{\text {fibre }}$. Considering that the microscopic stiffness of most soft tissues ranges between $0.3-100 \mathrm{kPa}$ [29], this suggests that electrospun networks with $E^{\text {fibre }}>100 \mathrm{MPa}$ might provide an excessively stiff micro-environment to adhering cells. In our analysis, substrates were approximately matched in terms of macroscopic stiffness. Vice versa, due to the mentioned proportionality, we note that matching the fibre modulus $E^{\text {fibre }}$, would affect the magnitude of the cell work, i.e. result in a $\sim 30 \%$ decrease for the $0.3 \mu \mathrm{m}$ networks, or a corresponding increase for the networks with $d_{\mathrm{F}}=1 \mu \mathrm{m}$. The potential impact of matching of network kinematics in terms of lateral contraction (Fig. 1b) is more difficult to assess, since the needed changes of topological parameters would also affect the micro- and macroscopic stiffness with different magnitude.

The predicted ratios of microscopic to macroscopic stiffness are significantly lower than suggested by experimental measurements in native tissues, which are in typical ranges of $\sim 10^{-2}-10^{-3}$ (cf. [29]). This points to a still significantly stiffer micro-environment for cells on an electrospun scaffold with comparable macroscopic stiffness than the native tissue. Yet, the networks analysed in Sec. 2.2 only represent two possible configurations within the wide spectrum, and higher ratios may be achieved by other combinations of the governing network parameters, in particular fibre diameter $d_{\mathrm{F}}$, mean segment length $\bar{l}_{\mathrm{S}}$, and average curvature radius $\bar{r}$. Therefore, these parameters were varied individually to study their effect on the length scale dependent stiffness. The impact of the network topology on the multiscale stiffness factor (Fig. 7c) may be explained by different fibre deformation modes that characterize the decoupling of stiffness over the length scales: While the macroscopic tensile stiffness mainly originates from the tensile loading of all straightened fibres (parallel arrangement), the stiffness at cell scale results from contributions of both stretched and bent fibres. This makes the ratio between bending and tensile stiffness a particularly important characteristic, which is strongly governed by, and grows quadratically with the fibre diameter. In summary, the parametric studies demonstrate that the multiscale stiffness ratio (Fig. 7c) can be altered over orders of magnitude by tuning the topological parameters, thus highlighting the broad design spectrum available for tuning the mechanical biocompatibility of such materials. At the same time, it emphasises once again the limited information contained in the stiffness values obtained in macroscopic tensile tests (Fig. 7a), since these may be the result of very different sets of topological parameters, and thus micromechanical environments (Fig. 7b).

\subsection{The effect of macroscopic deformations}

The analysis of fibre topology in stretched networks suggests that the microscopic stiffening correlates with the fibre slackness in the network. While in the unloaded configuration the initial fibre curvature introduces some slackness and thus a bending dominated early phase of deformation, fibres straighten upon macroscopic deformation. For 
the networks with $d_{\mathrm{F}}=1.0 \mu \mathrm{m}$, corresponding analyses indicate that the fibres finally become taught for strains $>3 \%$ (Fig. 9). Whereas equibiaxial loadings finally straightens all fibres, uniaxial loading is always associated with a large part of curled fibres due to the large lateral contraction exhibited by the networks. This finding also rationalizes the dependence of the magnitude of microscopic stiffening depending on the macroscopic loading observed in these networks (Fig. 8). The threshold strain for fibre straightening and network stiffening observed for the networks with $d_{\mathrm{F}}=1.0 \mu \mathrm{m}(\sim 3 \%)$ is very close to the fibre slackness strain of $2.6 \%$ determined analytically from the corresponding sinusoidal shape as the ratio of the contour length of one period over the wavelength. For the networks with $d_{\mathrm{F}}=0.3 \mu \mathrm{m}$, the analogously determined slackness strain of $10.3 \%$ indicates that these networks only become taught for higher deformations. This might explains the very low fraction of straightened fibres (Fig. 9) and the modest stiffness increase (Fig. 8) observed in the analyzed strain range or these networks.

a)

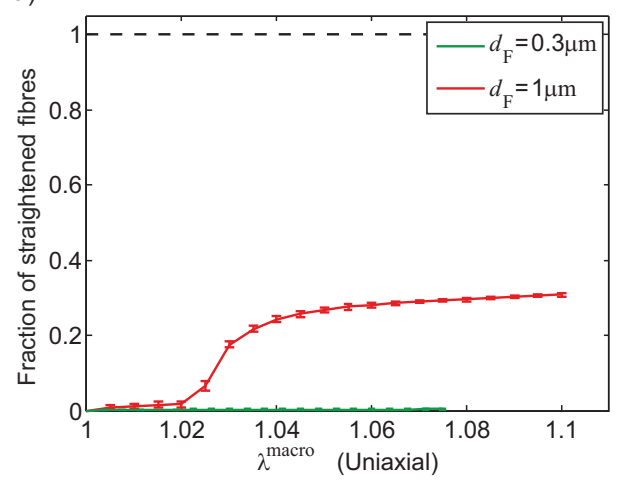

b)

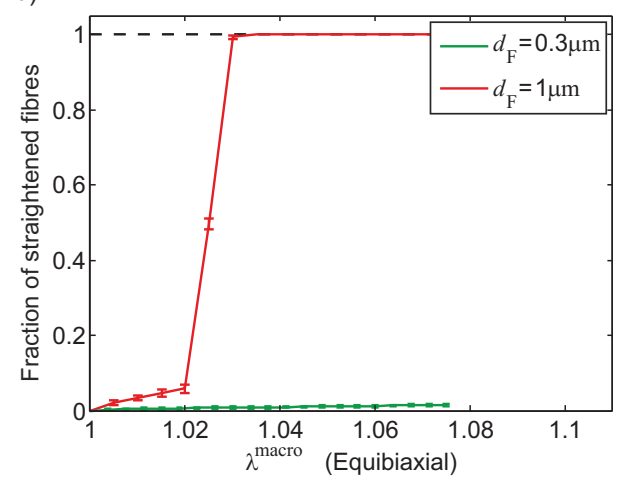

Figure 9: Fraction of straightened fibres within networks with macroscopically with macroscopically applied uniaxial (a) and equibiaxial tensile loadings (b). A fibre is identified as straightened when its end-to-end length is greater than its contour length in the undeformed configuration. Data is reported as mean and standard deviation for $N=10$ networks.

Since macroscopically induced microscopic stiffening originates in the topology of the networks, it is not expected to occur in homogeneous substrates with fairly linear response. Considering that this stiffening provides a cue for cellular mechanotransduction, this highlights another important difference between homogeneous and microstructured cell substrates. In [16] we have identified challenges associated with the prediction of the implant-tissue behaviour in-vivo. Little is known about the presence and magnitude of microscopic stiffening mechanisms in native tissues. It is thus speculation whether ESNs could be designed that feature similar microscopic stiffening, and that would provide mechanical biocompatibility with regard to this aspect. However, our numerical studies clearly demonstrate that it is not sufficient to match the native mechanical environment of cells in one static condition, but probably the whole range of expected physiological deformations needs to be considered (cf. [16]).

\subsection{Transferability of results and limitations}

Electrospun networks are treated as promising candidates for tissue engineering applications [6], also - or in particular - because they provide a fibrous environment to cells that resembles the ECM of native tissues. In many details, however, these networks differ. On the network scale, ESNs are distinguished by very long, often nearly straight fibres that form scissor-like or welded cross-links of coordination 4 [63,94], and are deposited in layers with typically very little out-of-plane depth. Conversely, collagen networks appear in a wide variety of morphologies, the structure of cross-links is much more disperse. On the level of single fibre elements, collagen fibres and their bundles in some soft tissues are indeed of comparable diameter as electrospun fibres, but the latter are composed of single fibrils furnishing them with bending, compressive and shear behaviour very different from bulk polymer fibres [95]. Moreover, the present study considered cells on the surface of fibrous substrates. While this may be representative for specific experimental configurations used in mechanobiological investigations and some types of cells, e.g. epithelial cells, in their native environment the majority of cells are surrounded by a three dimensional ECM. A transfer of the here obtained quantitative results to native tissues is thus questionable. Nevertheless, some of 
the results presented in this work point to general characteristics of network-like materials, including soft biological tissues. This concerns at the first instance (i) the length-scale dependence of material properties that, in fact, is well known in tissue biomechanics with tissue moduli spanning orders of magnitude [29]. In this regard, our unbiased computational simulations, that demonstrate the decoupling of stiffness as an inevitable multiscale property, help reconciliating these apparently controversial results. Further evidence that supports the applicability of our results to native tissues can be deduced from the many studies that document strain amplifications or attenuations in tissues over the scales $[24,27,96]$ and that thus point to scale-dependent deformation mechanisms. It is furthermore evident that any type of random network, including ESNs and collagenous ECM, will offer (ii) a broad spectrum of stiffness values at the anchoring points for a cell. Depending on the ratio between network and cell length scales, this heterogeneity may vanish when averaged over a cell, so that (iii) cells as a whole within networks may still experience reasonably homogeneous mechanical cues. The (iv) long-range transmission of cell-induced deformations (Fig. 4b) was shown to be only weakly dependent on fibre topology, and thus appears as another property transferable to collagenous networks. Finally, since macroscopic deformation changes the microscopic environment through a deformation and rotation of fibre segments, it necessarily changes the cell environment so that (v) microscopic stiffness will depend on the macroscopic state of deformation. With regard to soft tissue characterisation, this seems particularly relevant since the choice of configuration in which microscopic tests such as indentation are performed, e.g. in-vivo, unloaded or pre-strained, may strongly affect the result.

Although our fibre network model for the prediction of the mechanical behaviour of ESNs is among the most dedicated approaches, it is based on several simplifications that may affect the results. For example, fibre diameters in ESNs are typically non-uniform but distributed around a mean. We have recently investigated the impact of including these distributions in computational models and found that the macroscopic response was marginally affected [97]. On the contrary, it is expected that a dispersion of fibre diameters would increase the spatial heterogeneity of the cell felt stiffness, and thus change to some extent the average local stiffness, due to the non-linear relation between fibre diameter and fibre bending stiffness. Since such variability of $d_{\mathrm{F}}$ would affect both networks in a similar, albeit not entirely equal manner, the general results and conclusions obtained in this study with uniform $d_{\mathrm{F}}$ are expected to hold even for diameters distributed around corresponding mean values.

Similarly, simplifications were made for the modelling the cell and its interaction with the substrate. The model considers the cell quasi-circular, with 24 focal adhesions (or clusters) that have equal distance of $15 \mu \mathrm{m}$ from the cell's centre. While the numbers are motivated by experimental data [79], the quasi-circular shape is clearly only one of many possibilities, and is probably little representative for polarised cells. However, it is well-defined and advantageous as regards the mechanical analysis since (i) radial forces at sites distributed along the circumference lead to a force field that directly satisfies force and moment equilibrium, and (ii) the same radial displacement of each adhesion site leads to an equal amount of global strain in radial direction, thus the stiffness at each site can be directly computed from the corresponding force. The adhesion sites were selected independent of local mechanical properties, whereas real cells test their environment, and typically form stable focal adhesions only at sites that provide sufficient mechanical resistance, while too compliant substrate response leads to focal diffuse and dynamic adhesion assembly [98]. In view of this, the model might be particularly representative for the phase of isotropic cell spreading, where the cell steadily increases its projected area but maintains a quasi-circular shape [99]. Also the quantitative results provided in this study might depend on the used modelling simplifications. To quantify cell work, the model assumes that all focal adhesions are displaced by the same amount. Relaxing this assumption would probably affect the total amount of cellular work. However, considering the number of active focal adhesions independent of the substrate, the assumption leads to a scalar measure which is directly comparable between load cases and substrates. Similarly, its comparison with values predicted for homogeneous substrates is expected to result in a robust measure since both models apply the same assumption, therefore substantiating the relevance of the proposed multiscale stiffness factor. This argument of comparability also applies to the chosen cell size, which has also been found to influence the microscopic stiffness to some extent, where the number of FAs was held constant (data not shown). However, since this number typically changes with cell size (cf. [79]) this result is difficult to assess, and emphasizes the fact that the present study analyses specific cell-network combinations.

While the study focuses on the effect of fibre diameter, curvature and segment length on ESN multiscale stiffness, other morphological ESN parameters are known to affect the mechanobiological response of adhering cells, such as porosity and pore shape [53] or anisotropy, i.e. non-uniform fibre orientation [48, 51, 52]. While the model could be extended accordingly to perform detailed studies, we note that the pore size is related to segment length [63], 
and that changes in cell shape and alignment have been associated with both initial alignment and deformationinduced reorientation of fibres $[42,100]$. The latter case suggests a combination of passive deformation with an active mechanotransductive response of the cells. The passive part can be captured by our current model (cf. Sec. 4.3), i.e. the model cell can identify the anisotropy, but does not respond actively.

\section{Conclusions}

This study is dedicated to the mechanical environment of cells interacting with the surface of electrospun tissue engineering scaffolds in order to quantify metrics that allow assessing the mechanical biocompatibility of these engineered fabrics on macroscopic and microscopic length scales. The results suggest that, similar to biopolymer networks, deformations induced by cellular contraction are transmitted over long ranges in ESNs, and thus affect much larger regions than on a homogeneous substrate with comparable macroscopic stiffness. The network's structural stiffness experienced by the cells at individual attachment sites varies tremendously, but this heterogeneity decreases substantially when averaging over the cell. This raises the important question, whether the signals from single FAs or their average is more important for the cell's mechanosensitive capabilities. While our results show that the cell scale is already sufficient to obtain a fairly homogenised signal, the study cannot answer this question, and we emphasise that a cell might additionally be able to detach, and to reattach to favourable network sites in order to actively modulate the sensed stiffness.

The elastic modulus of the fibres, the cell-scale stiffness and macro-scale modulus of the ESN are largely decoupled. Therefore, in contrast to continuous homogeneous substrates, ESNs with comparable macroscopic stiffness can have a wide range of cell-scale stiffness, with orders of magnitude in between. The stiffness ratio strongly depends on fibre topology and network morphology, thus offering means to tune macro- and micromechanics independently to satisfy given requirements on the two scales. In principle, this allows fulfilling the microscopical mechanical function (suitable macroscopic stiffness and strength) as well as providing the micromechanical compliance and specific local deformation mechanisms at cell lengthscale. Nevertheless, despite this wide achievable range, the average forces required to elicit cell-typical displacements in the electrospun scaffolds with typical properties investigated here, exceed the common cell tractions measured in traction force microscopy. Accordingly, our results suggest that for cells, most ESNs, with $E^{\text {fibre }}$ typically in the range of several hundred MPa, will rather feel like stiff or even rigid.

Finally, we have shown that the transfer of loads over the scales depends on the state of deformation, owing to the discrete structure of the network and the non-affine motion of its constituents. As a consequence, the ratio between macroscopic and cell-scale stiffness is not a constant but might change considerably. These findings point to yet another dimension of the already complex task of mechanically biocompatible scaffold design: The engineered materials have to match the multi-mechanical behaviour of their native templates not in one but all physiologically relevant states. In summary, our results show that ESNs capture some of the salient multiscale mechanical characteristics of soft biological tissues, at least qualitatively, including long range interactions within the network and largely decoupled stiffnesses measured at different length scales. Moreover, the study revealed some important parameters that affect these characteristics, such as fibre diameter and average curvature radius. The work thus highlights that computational methods can be used during the process of scaffold design and analysis to guide the development, particularly because they provide access to essential data that are extremely difficult to collect in experiments.

\section{Acknowledgements}

This work was supported by the Swiss National Science Foundation (grant number 155918). 


\section{Appendix A. Modelling of the interaction of cells with homogeneous substrates}
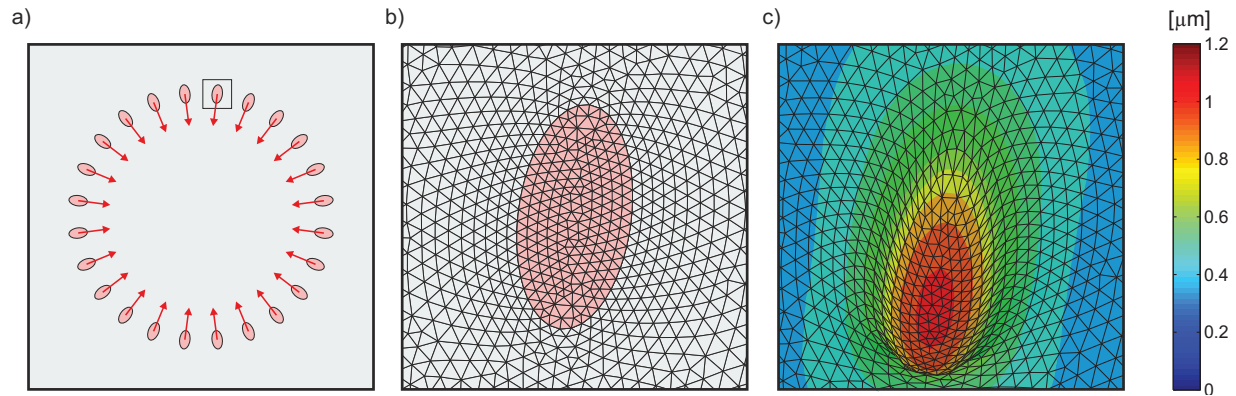

Figure A.1: a) Application of cell traction stresses on the surface of a homogeneous substrate. b) Detailed view of mesh discretization in the region around the focal adhesion indicated in a). c) Localized surface displacement field induced by cell tractions in the substrate.

\section{Appendix B. Network size dependence on cell-felt stiffness}

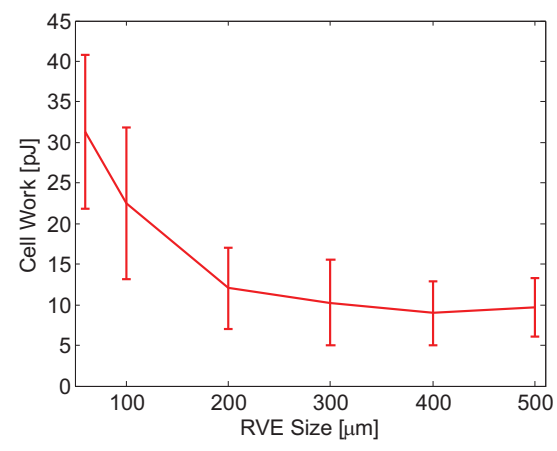

Figure B.1: Dependence of RVE size (length and width) for the cell-network interaction studies. The reported cell work $W^{\text {Cell }}$ evaluated at $\varepsilon^{\mathrm{A}}=10 \%$ on $N=20$ networks with $d_{\mathrm{F}}=1.0 \mu \mathrm{m}$ stabilizes around $200 \mu \mathrm{m}$.

\section{References}

[1] D. Li, Y. Xia, Electrospinning of nanofibers: Reinventing the wheel?, Advanced Materials 16 (2004) 1151-1170.

[2] A. Greiner, J. H. Wendorff, Electrospinning: A fascinating method for the preparation of ultrathin fibers, Angewandte Chemie International Edition 46 (2007) 5670-5703.

[3] J. Fang, H. Niu, T. Lin, X. Wang, Applications of electrospun nanofibers, Chinese Science Bulletin 53 (2008) $2265-2286$.

[4] T. J. Sill, H. A. von Recum, Electrospinning: Applications in drug delivery and tissue engineering, Biomaterials 29 (2008) $1989-2006$.

[5] R. Sridhar, R. Lakshminarayanan, K. Madhaiyan, V. Amutha Barathi, K. H. C. Lim, S. Ramakrishna, Electrosprayed nanoparticles and electrospun nanofibers based on natural materials: Applications in tissue regeneration, drug delivery and pharmaceuticals, Chemical Society Reviews 44 (2015) 790-814.

[6] S. Agarwal, J. H. Wendorff, A. Greiner, Use of electrospinning technique for biomedical applications, Polymer 49 (2008) 5603-5621.

[7] M. Norouzi, S. M. Boroujeni, N. Omidvarkordshouli, M. Soleimani, Advances in skin regeneration: Application of electrospun scaffolds, Advanced Healthcare Materials 4 (2015) 1114-1133.

[8] M. Kitsara, O. Agbulut, D. Kontziampasis, Y. Chen, P. Menasché, Fibers for hearts: A critical review on electrospinning for cardiac tissue engineering, Acta Biomaterialia 48 (2017) 20-40.

[9] A. Hasan, A. Memic, N. Annabi, M. Hossain, A. Paul, M. R. Dokmeci, F. Dehghani, A. Khademhosseini, Electrospun scaffolds for tissue engineering of vascular grafts, Acta Biomaterialia 10 (2014) 11-25. 
[10] R. Skalak, Tissue engineering, in: Proceedings of the 15th Annual International Conference of the IEEE Engineering in Medicine and Biology Society, 1993, pp. 1112-1113. doi:10.1109/IEMBS.1993.979045.

[11] R. Langer, J. Vacanti, Tissue engineering, Science 260 (1993) 920-926.

[12] R. M. Nerem, A. Sambanis, Tissue engineering: From biology to biological substitutes, Tissue engineering 1 (1995) 3-13.

[13] J. P. Vacanti, R. Langer, Tissue engineering: The design and fabrication of living replacement devices for surgical reconstruction and transplantation, The lancet 354 (1999) 32-34.

[14] D. Williams, Concepts in biocompatibility: New biomaterials, new paradigms and new testing regimes, in: Biocompatibility and performance of medical devices, 2012, pp. 3-17. doi:10.1533/9780857096456.1.1.

[15] J. C. Rose, L. De Laporte, Hierarchical design of tissue regenerative constructs, Advanced Healthcare Materials 7 (2017) 1701067.

[16] E. Mazza, A. E. Ehret, Mechanical biocompatibility of highly deformable biomedical materials, Journal of the Mechanical Behavior of Biomedical Materials 48 (2015) 100-124.

[17] D. L. Butler, S. A. Goldstein, F. Guilak, Functional tissue engineering: The role of biomechanics, Journal of Biomechanical Engineering 20 (2000) 570-575.

[18] S. Pal, Mechanical properties of biological materials, in: Design of Artificial Human Joints \& Organs, 2014, pp. 23-40. doi:10.1007/9781-4614-6255-2_2.

[19] G. Charras, E. Sahai, Physical influences of the extracellular environment on cell migration, Nature Reviews Molecular Cell Biology 15 (2014) 813-824.

[20] K. Ghosh, D. E. Ingber, Micromechanical control of cell and tissue development: Implications for tissue engineering, Advanced Drug Delivery Reviews 59 (2007) 1306 - 1318.

[21] B. J. Briscoe, L. Fiori, E. Pelillo, Nano-indentation of polymeric surfaces, Journal of Physics D: Applied Physics 31 (1998) 2395.

[22] D. M. Ebenstein, L. A. Pruitt, Nanoindentation of biological materials, Nano Today 1 (2006) 26-33.

[23] T. Saxena, J. L. Gilbert, J. M. Hasenwinkel, A versatile mesoindentation system to evaluate the micromechanical properties of soft, hydrated substrates on a cellular scale, Journal of Biomedical Materials Research Part A 90A (2009) 1206-1217.

[24] W. M. Han, S.-J. Heo, T. P. Driscoll, L. J. Smith, R. L. Mauck, D. M. Elliott, Macro- to microscale strain transfer in fibrous tissues is heterogeneous and tissue-specific, Biophysical Journal 105 (2013) 807-817.

[25] C. Jayyosi, G. Fargier, M. Coret, K. Bruyère-Garnier, Photobleaching as a tool to measure the local strain field in fibrous membranes of connective tissues, Acta Biomaterialia 10 (2014) 2591-2601.

[26] A. Anssari-Benam, H. S. Gupta, H. R. Screen, Strain transfer through the aortic valve, Journal of biomechanical engineering 134 (2012) 061003.

[27] B. Bell, E. Nauman, S. Voytik-Harbin, Multiscale strain analysis of tissue equivalents using a custom-designed biaxial testing device, Biophysical Journal 102 (2012) 1303-1312.

[28] X. Ma, M. E. Schickel, M. D. Stevenson, A. L. Sarang-Sieminski, K. J. Gooch, S. N. Ghadiali, R. T. Hart, Fibers in the extracellular matrix enable long-range stress transmission between cells, Biophysical Journal 104 (2013) 1410-1418.

[29] C. T. McKee, J. A. Last, P. Russell, C. J. Murphy, Indentation versus tensile measurements of Young's modulus for soft biological tissues, Tissue Engineering Part B: Reviews 17 (2011) 155-164.

[30] G. Clermont, Non-clinical functional evaluation of medical devices: General recommendations and examples for soft tissue implants, in: Biocompatibility and Performance of Medical Devices, 2012, pp. 345-361. doi:10.1533/9780857096456.3.345.

[31] J. H.-C. Wang, J.-S. Lin, Cell traction force and measurement methods, Biomechanics and Modeling in Mechanobiology 6 (2007) 361.

[32] U. S. Schwarz, J. R. Soiné, Traction force microscopy on soft elastic substrates: A guide to recent computational advances, Biochimica et Biophysica Acta - Molecular Cell Research 1853 (2015) 3095-3104.

[33] P. Roca-Cusachs, V. Conte, X. Trepat, Quantifying forces in cell biology, Nature Cell Biology 19 (2017) 742-751.

[34] P. A. Janmey, D. A. Weitz, Dealing with mechanics: Mechanisms of force transduction in cells, Trends in Biochemical Sciences 29 (2004) 364-370.

[35] D. E. Ingber, Cellular mechanotransduction: Putting all the pieces together again, The FASEB Journal 20 (2006) 811-827.

[36] S.-Y. Tee, J. Fu, C. S. Chen, P. A. Janmey, Cell shape and substrate rigidity both regulate cell stiffness, Biophysical Journal 100 (2011) 25-27.

[37] T. Yeung, P. C. Georges, L. A. Flanagan, B. Marg, M. Ortiz, M. Funaki, N. Zahir, W. Ming, V. Weaver, P. A. Janmey, Effects of substrate stiffness on cell morphology, cytoskeletal structure, and adhesion, Cell Motility and the Cytoskeleton 60 (2005) 24-34.

[38] C.-M. Lo, H.-B. Wang, M. Dembo, Y. li Wang, Cell movement is guided by the rigidity of the substrate, Biophysical Journal 79 (2000) $144-152$.

[39] C. J. Bettinger, R. Langer, J. T. Borenstein, Engineering substrate topography at the micro- and nanoscale to control cell function, Angewandte Chemie International Edition 48 (2009) 5406-5415.

[40] M. Nikkhah, F. Edalat, S. Manoucheri, A. Khademhosseini, Engineering microscale topographies to control the cell-substrate interface, Biomaterials 33 (2012) 5230-5246.

[41] N. Caille, Y. Tardy, J. J. Meister, Assessment of strain field in endothelial cells subjected to uniaxial deformation of their substrate, Annals of Biomedical Engineering 26 (1998) 409-416.

[42] J. H.-C. Wang, P. Goldschmidt-Clermont, J. Wille, F. C.-P. Yin, Specificity of endothelial cell reorientation in response to cyclic mechanical stretching, Journal of Biomechanics 34 (2001) 1563-1572.

[43] C. Galbraith, R. Skalak, S. Chien, Shear stress induces spatial reorganization of the endothelial cell cytoskeleton, Cell Motility and the Cytoskeleton 40 (1998) 317-330.

[44] A. D. Acevedo, S. S. Bowser, M. E. Gerritsen, R. Bizios, Morphological and proliferative responses of endothelial cells to hydrostatic pressure: Role of fibroblast growth factor, Journal of Cellular Physiology 157 (1993) 603-614.

[45] K. M. Kennedy, A. Bhaw-Luximon, D. Jhurry, Cell-matrix mechanical interaction in electrospun polymeric scaffolds for tissue engineering: Implications for scaffold design and performance, Acta Biomaterialia 50 (2017) 41-55.

[46] I. K. Kwon, S. Kidoaki, T. Matsuda, Electrospun nano- to microfiber fabrics made of biodegradable copolyesters: Structural characteristics, 
mechanical properties and cell adhesion potential, Biomaterials 26 (2005) 3929-3939.

[47] A. S. Badami, M. R. Kreke, M. S. Thompson, J. S. Riffle, A. S. Goldstein, Effect of fiber diameter on spreading, proliferation, and differentiation of osteoblastic cells on electrospun poly(lactic acid) substrates, Biomaterials 27 (2006) 596-606.

[48] C. A. Bashur, R. D. Shaffer, L. A. Dahlgren, S. A. Guelcher, A. S. Goldstein, Effect of fiber diameter and alignment of electrospun polyurethane meshes on mesenchymal progenitor cells, Tissue Engineering Part A 15 (2009) 2435-2445.

[49] G. T. Christopherson, H. Song, H.-Q. Mao, The influence of fiber diameter of electrospun substrates on neural stem cell differentiation and proliferation, Biomaterials 30 (2009) 556-564.

[50] E. S. Fioretta, M. Simonet, A. I. P. M. Smits, F. P. T. Baaijens, C. V. C. Bouten, Differential response of endothelial and endothelial colony forming cells on electrospun scaffolds with distinct microfiber diameters, Biomacromolecules 15 (2014) 821-829.

[51] F. Yang, R. Murugan, S. Wang, S. Ramakrishna, Electrospinning of nano/micro scale poly(l-lactic acid) aligned fibers and their potential in neural tissue engineering, Biomaterials 26 (2005) 2603-2610.

[52] S. Y. Chew, R. Mi, A. Hoke, K. W. Leong, The effect of the alignment of electrospun fibrous scaffolds on schwann cell maturation, Biomaterials 29 (2008) 653-661.

[53] J. L. Lowery, N. Datta, G. C. Rutledge, Effect of fiber diameter, pore size and seeding method on growth of human dermal fibroblasts in electrospun poly ( $\epsilon$-caprolactone) fibrous mats, Biomaterials 31 (2010) 491-504.

[54] E. Vatankhah, M. P. Prabhakaran, D. Semnani, S. Razavi, M. Morshed, S. Ramakrishna, Electrospun tecophilic/gelatin nanofibers with potential for small diameter blood vessel tissue engineering, Biopolymers 101 (2014) 1165-1180.

[55] K. Wingate, W. Bonani, Y. Tan, S. Bryant, W. Tan, Compressive elasticity of three-dimensional nanofiber matrix directs mesenchymal stem cell differentiation to vascular cells with endothelial or smooth muscle cell markers, Acta Biomaterialia 8 (2012) $1440-1449$.

[56] B. M. Baker, B. Trappmann, W. Y. Wang, M. S. Sakar, I. L. Kim, V. B. Shenoy, J. A. Burdick, C. S. Chen, Cell-mediated fibre recruitment drives extracellular matrix mechanosensing in engineered fibrillar microenvironments, Nature Materials 14 (2015) $1262-1268$.

[57] J. P. Marquez, G. M. Genin, G. I. Zahalak, E. L. Elson, Thin bio-artificial tissues in plane stress: The relationship between cell and tissue strain, and an improved constitutive model, Biophysical Journal 88 (2005) 765-777.

[58] V. K. Lai, M. F. Hadi, R. T. Tranquillo, V. H. Barocas, A multiscale approach to modeling the passive mechanical contribution of cells in tissues, Journal of biomechanical engineering 135 (2013) 071007.

[59] M. S. Rudnicki, H. A. Cirka, M. Aghvami, E. A. Sander, Q. Wen, K. L. Billiar, Nonlinear strain stiffening is not sufficient to explain how far cells can feel on fibrous protein gels, Biophysical Journal 105 (2013) 11-20.

[60] H. Wang, A. Abhilash, C. S. Chen, R. G. Wells, V. B. Shenoy, Long-range force transmission in fibrous matrices enabled by tension-driven alignment of fibers, Biophysical Journal 107 (2014) 2592-2603.

[61] S. G. Kumbar, S. P. Nukavarapu, R. James, L. S. Nair, C. T. Laurencin, Electrospun poly(lactic acid-co-glycolic acid) scaffolds for skin tissue engineering, Biomaterials 29 (2008) 4100-4107.

[62] M.-C. Kim, Y. R. Silberberg, R. Abeyaratne, R. D. Kamm, H. H. Asada, Computational modeling of three-dimensional ECM-rigidity sensing to guide directed cell migration, Proceedings of the National Academy of Sciences 115 (2018) E390-E399.

[63] M. Zündel, E. Mazza, A. E. Ehret, A 2.5D approach to the mechanics of electrospun fibre mats, Soft Matter 13 (2017) 6407-6421.

[64] V. Kouznetsova, W. A. M. Brekelmans, F. P. T. Baaijens, An approach to micro-macro modeling of heterogeneous materials, Computational Mechanics 27 (2001) 37-48.

[65] T. Stylianopoulos, V. H. Barocas, Volume-averaging theory for the study of the mechanics of collagen networks, Computer Methods in Applied Mechanics and Engineering 196 (2007) 2981-2990.

[66] R. Y. Dhume, V. H. Barocas, Fiber-network modeling in biomechanics: Theoretical and analytical approaches, in: Biomechanics: Trends in Modeling and Simulation, 2017, pp. 271-307. doi:10.1007/978-3-319-41475-1_7.

[67] A. Cipitria, A. Skelton, T. R. Dargaville, P. D. Dalton, D. W. Hutmacher, Design, fabrication and characterization of pcl electrospun scaffolds - A review, J. Mater. Chem. 21 (2011) 9419-9453.

[68] S. Soliman, S. Sant, J. W. Nichol, M. Khabiry, E. Traversa, A. Khademhosseini, Controlling the porosity of fibrous scaffolds by modulating the fiber diameter and packing density, Journal of Biomedical Materials Research Part A 96 (2011) 566-574.

[69] J. Rnjak-Kovacina, A. S. Weiss, Increasing the pore size of electrospun scaffolds, Tissue Engineering Part B: Reviews 17 (2011) $365-372$.

[70] D. Gupta, J. Venugopal, M. P. Prabhakaran, V. G. Dev, S. Low, A. T. Choon, S. Ramakrishna, Aligned and random nanofibrous substrate for the in vitro culture of schwann cells for neural tissue engineering, Acta Biomaterialia 5 (2009) 2560-2569.

[71] L. Ye, X. Wu, Q. Mu, B. Chen, Y. Duan, X. Geng, Y. Gu, A. Zhang, J. Zhang, Z. guo Feng, Heparin-conjugated pcl scaffolds fabricated by electrospinning and loaded with fibroblast growth factor 2, Journal of Biomaterials Science, Polymer Edition 22 (2011) $389-406$.

[72] Q. P. Pham, U. Sharma, A. G. Mikos, Electrospun poly( $\epsilon$-caprolactone) microfiber and multilayer nanofiber/microfiber scaffolds: Characterization of scaffolds and measurement of cellular infiltration, Biomacromolecules 7 (2006) 2796-2805.

[73] C.-L. Pai, M. Boyce, G. Rutledge, On the importance of fiber curvature to the elastic moduli of electrospun nonwoven fiber meshes, Polymer 52 (2011) 6126-6133.

[74] S.-C. Wong, A. Baji, S. Leng, Effect of fiber diameter on tensile properties of electrospun poly( $€$-caprolactone), Polymer 49 (2008) 4713-4722.

[75] K. L. Alderson, A. Alderson, K. E. Evans, The interpretation of the strain-dependent poisson's ratio in auxetic polyethylene, The Journal of Strain Analysis for Engineering Design 32 (1997) 201-212.

[76] K. Bircher, A. E. Ehret, E. Mazza, Mechanical characteristics of bovine Glisson's capsule as a model tissue for soft collagenous membranes, Journal of biomechanical engineering 138 (2016) 081005

[77] J. Hewitt, F. Guilak, R. Glisson, T. P. Vail, Regional material properties of the human hip joint capsule ligaments, Journal of Orthopaedic Research 19 (2001) $359-364$

[78] H. A. Lynch, W. Johannessen, J. P. Wu, A. Jawa, D. M. Elliott, Effect of fiber orientation and strain rate on the nonlinear uniaxial tensile material properties of tendon, Journal of biomechanical engineering 125 (2003) 726-731.

[79] J. Fu, Y.-K. Wang, M. T. Yang, R. A. Desai, X. Yu, Z. Liu, C. S. Chen, Mechanical regulation of cell function with geometrically modulated elastomeric substrates, Nature Methods 7 (2010) 733-736. 
[80] M. Zündel, A. E. Ehret, E. Mazza, Factors influencing the determination of cell traction forces, PLOS ONE 12 (2017) 1-18.

[81] J. P. Winer, S. Oake, P. A. Janmey, Non-linear elasticity of extracellular matrices enables contractile cells to communicate local position and orientation, PLOS ONE 4 (2009) 1-11.

[82] W. S. Leong, C. Y. Tay, H. Yu, A. Li, S. C. Wu, D.-H. Duc, C. T. Lim, L. P. Tan, Thickness sensing of hMSCs on collagen gel directs stem cell fate, Biochemical and Biophysical Research Communications 401 (2010) 287-292.

[83] C. T. Koh, M. L. Oyen, Toughening in electrospun fibrous scaffolds, APL Materials 3 (2015) 014908.

[84] A. E. Ehret, K. Bircher, A. Stracuzzi, V. Marina, M. Zündel, E. Mazza, Inverse poroelasticity as a fundamental mechanism in biomechanics and mechanobiology, Nature Communications 8 (2017) 1002.

[85] M. Ghibaudo, A. Saez, L. Trichet, A. Xayaphoummine, J. Browaeys, P. Silberzan, A. Buguin, B. Ladoux, Traction forces and rigidity sensing regulate cell functions, Soft Matter 4 (2008) 1836-1843.

[86] V. F. Achterberg, L. Buscemi, H. Diekmann, J. Smith-Clerc, H. Schwengler, J.-J. Meister, H. Wenck, S. Gallinat, B. Hinz, The nano-scale mechanical properties of the extracellular matrix regulate dermal fibroblast function, Journal of Investigative Dermatology 134 (2014) 1862-1872.

[87] C. Ciobanasu, B. Faivre, C. L. Clainche, Integrating actin dynamics, mechanotransduction and integrin activation: The multiple functions of actin binding proteins in focal adhesions, European Journal of Cell Biology 92 (2013) 339 - 348. SPP 1464:Actin Dynamics Part I.

[88] N. Wang, Review of cellular mechanotransduction, Journal of Physics D: Applied Physics 50 (2017) 233002.

[89] A. J. Maniotis, C. S. Chen, D. E. Ingber, Demonstration of mechanical connections between integrins, cytoskeletal filaments, and nucleoplasm that stabilize nuclear structure, Proceedings of the National Academy of Sciences 94 (1997) 849-854.

[90] T. P. Driscoll, B. D. Cosgrove, S.-J. Heo, Z. E. Shurden, R. L. Mauck, Cytoskeletal to nuclear strain transfer regulates YAP signaling in mesenchymal stem cells, Biophysical Journal 108 (2015) 2783-2793.

[91] R. Picu, S. Deogekar, M. Islam, Poisson's contraction and fiber kinematics in tissue: Insight from collagen network simulations, Journal of biomechanical engineering 140 (2018) 021002.

[92] V. Thomas, M. V. Jose, S. Chowdhury, J. F. Sullivan, D. R. Dean, Y. K. Vohra, Mechano-morphological studies of aligned nanofibrous scaffolds of polycaprolactone fabricated by electrospinning, Journal of Biomaterials Science, Polymer Edition 17 (2006) $969-984$.

[93] K. Lee, H. Kim, M. Khil, Y. Ra, D. Lee, Characterization of nano-structured poly( $\varepsilon$-caprolactone) nonwoven mats via electrospinning, Polymer 44 (2003) 1287-1294.

[94] R. C. Picu, Mechanics of random fiber networks - A review, Soft Matter 7 (2011) 6768-6785.

[95] K. Bircher, A. E. Ehret, E. Mazza, Microstructure based prediction of the deformation behavior of soft collagenous membranes, Soft Matter 13 (2017) 5107-5116.

[96] A. D. Lewinsohn, A. Anssari-Benham, D. A. Lee, P. M. Taylor, A. H. Chester, M. H. Yacoub, H. R. C. Screen, Anisotropic strain transfer through the aortic valve and its relevance to the cellular mechanical environment, Proceedings of the Institution of Mechanical Engineers, Part H: Journal of Engineering in Medicine 225 (2011) 821-830.

[97] S. Domaschke, M. Zündel, E. Mazza, A. E. Ehret, A 3D computational model of electrospun networks and its application to inform a reduced modelling approach, International Journal of Solids and Structures (2018).

[98] R. J. Pelham, Y.-1. Wang, Cell locomotion and focal adhesions are regulated by substrate flexibility, Proceedings of the National Academy of Sciences 94 (1997) 13661-13665.

[99] Y. Xiong, P. Rangamani, M.-A. Fardin, A. Lipshtat, B. Dubin-Thaler, O. Rossier, M. P. Sheetz, R. Iyengar, Mechanisms controlling cell size and shape during isotropic cell spreading, Biophysical journal 98 (2010) 2136-2146.

[100] D. R. Nisbet, A. E. Rodda, D. I. Finkelstein, M. K. Horne, J. S. Forsythe, W. Shen, Surface and bulk characterisation of electrospun membranes: Problems and improvements, Colloids and Surfaces B: Biointerfaces 71 (2009) 1-12. 